\title{
Turbulence intermittency linked to the weakly coherent mode in ASDEX Upgrade I-mode plasmas
}

\author{
T. Happel,,${ }^{1, *}$ P. Manz, ${ }^{1,2}$ F. Ryter, ${ }^{1}$ P. Hennequin, ${ }^{3}$ A. Hetzenecker, ${ }^{1}$ G. D. Conway, ${ }^{1}$ \\ L. Guimarais, ${ }^{4}$ C. Honoré, ${ }^{3}$ U. Stroth, ${ }^{1,2}$ E. Viezzer, ${ }^{1}$ and the ASDEX Upgrade Team ${ }^{1}$ \\ ${ }^{1}$ Max Planck Institute for Plasma Physics, Boltzmannstr. 2, 85748 Garching, Germany \\ ${ }^{2}$ Physik-Department E28, Technische Universität München, James-Franck-Str. 1, 85748 Garching, Germany \\ ${ }^{3}$ Laboratoire de Physique des Plasmas, Ecole Polytechnique, 91128 Palaiseau, France \\ ${ }^{4}$ Instituto de Plasmas e Fusão Nuclear, Instituto Superior Técnico, Universidade de Lisboa, Portugal
}

(Dated: 2017-01-26)

\begin{abstract}
This paper shows for the first time a pronounced increase of extremely intermittent edge density turbulence behavior inside the confinement region related to the I-mode confinement regime in the ASDEX Upgrade tokamak. With improving confinement, the perpendicular propagation velocity of density fluctuations in the plasma edge increases together with the intermittency of the observed density bursts. Furthermore, it is shown that the weakly coherent mode, a fluctuation feature generally observed in I-mode plasmas, is connected to the observed bursts. It is suggested that the large amplitude density bursts could be generated by a non-linearity similar to that in the Korteweg-de-Vries equation which includes the radial temperature gradient.
\end{abstract}

PACS numbers: 52.35.Ra, 52.55.Fa, 52.70.Gw, 52.25.Os

On the way to obtaining energy through magnetic nuclear fusion, the discovery of an improved confinement regime called the high-confinement mode ( $\mathrm{H}$-mode), in contrast to the lower confinement L-mode, was a major achievement [1]. The H-mode is characterized by a quiescent plasma edge with steep gradients, also called transport barriers, in both the density and the temperature profiles. Generally, in transport barriers turbulence is reduced and steep gradients form in density and temperature, which results in a strong so-called pedestal in these quantities, leading to a better confinement. An alternate improved confinement regime is the so-called improved energy confinement regime (I-mode). Interestingly, it is characterized by a transport barrier in temperature only. Hence, energy transport behaves like in $\mathrm{H}$-mode $[2,3]$ while particle confinement is L-mode like. In I-mode, the edge-localized mode (ELM) does not develop [4, 5] and impurities do not accumulate due to the absence of a particle transport barrier [3]. The I-mode can only be obtained if the power threshold for $\mathrm{H}$ mode access is kept high, e.g. by using unfavorable magnetic configurations, and it is characterized by a quasi-coherent feature in the fluctuation characteristics called the weakly coherent mode (WCM) which resides in the plasma edge [6]. The WCM has been shown to be coupled to a geodesic acoustic mode (GAM), which explains the width of the WCM [7, 8]. The remaining unsolved issues regarding the nature of the Imode are: what is the underlying instability of the WCM? How can density and temperature fluctuations be decoupled to such a degree that a pedestal forms in one quantity and not the other?

This letter reports the observation of strongly intermittent density turbulence bursts in the edge confinement region of the ASDEX Upgrade tokamak (AUG). The results are directly related to the I-mode $[2,3]$ and may help to understand the decoupling of heat and particle transport. Moreover, since magnetically confined plasmas constitute, to first order, twodimensional (2D) turbulent systems which are still subject to the question of whether they can be intermittent [9-11], the observations add to the evidence of intermittency - often connected to self-organized criticality [12] - in quasi 2D turbulent systems [13-17]. In this context, it should be noted that substantial effort has been dedicated to the understanding of intermittency in the scrape-off layer of magnetically confined plasmas [18-23].

Experiments have been conducted on the ASDEX Upgrade tokamak [24]. The main diagnostic used in this work is a Doppler reflectometer $[25,26]$ measuring in the edge plasma region (last $1.5 \mathrm{~cm}$ inside the separatrix) and being sensitive to perpendicular wavenumbers of the turbulence in the range $k_{\perp}=11-13 \mathrm{~cm}^{-1}$. For the plasmas presented, this corresponds to $k_{\perp} \rho_{s} \approx 1.0-1.5$, where $\rho_{s}=\sqrt{m_{i} T_{e}} /(e B)$, with $m_{i}$ the ion mass, $T_{e}$ the electron temperature, $e$ the elementary charge and $B$ the magnetic field strength. The large variation in $k_{\perp} \rho_{s}$ compared to $k_{\perp}$ is due to the $T_{e}$ evolution during I-mode. First, to give a brief overview, a representative discharge is introduced and radial profiles of the perpendicular propagation velocity of density fluctuations during the confinement improvement are presented. Then, the density bursts and their connection to the WCM are described and a possible generation mechanism of the former is discussed.

Figure 1 shows time traces of several ASDEX Upgrade discharge parameters (AUG \#29741), as the plasma evolves from L-mode into I-mode confinement $(2.25 \mathrm{~s})$, which finally ends at the I-H transition (2.66 s). The discharge has a constant electron cyclotron resonance heating (ECRH) power of $740 \mathrm{~kW}$ (a). At $2.1 \mathrm{~s}$, one neutral beam injection (NBI) source with $2 \mathrm{MW}$ of heating power is added. Roughly $150 \pm 25 \mathrm{~ms}$ later, the transition into I-mode takes place, identified by the appearance of the WCM [27], which can be observed in the turbulence spectra of a conventional O-mode reflectometer [28] measuring at $\rho_{\text {pol }}=0.997$ in Figs. 1(e), (f) and (g). Here, $\rho_{\text {pol }}$ is the normalized poloidal flux radius. In the Lmode phase of the discharge, no WCM is visible (e). However, just after $2.25 \mathrm{~s}$, the WCM is observed (f). Later, in the well developed I-mode, the WCM is at slightly higher 


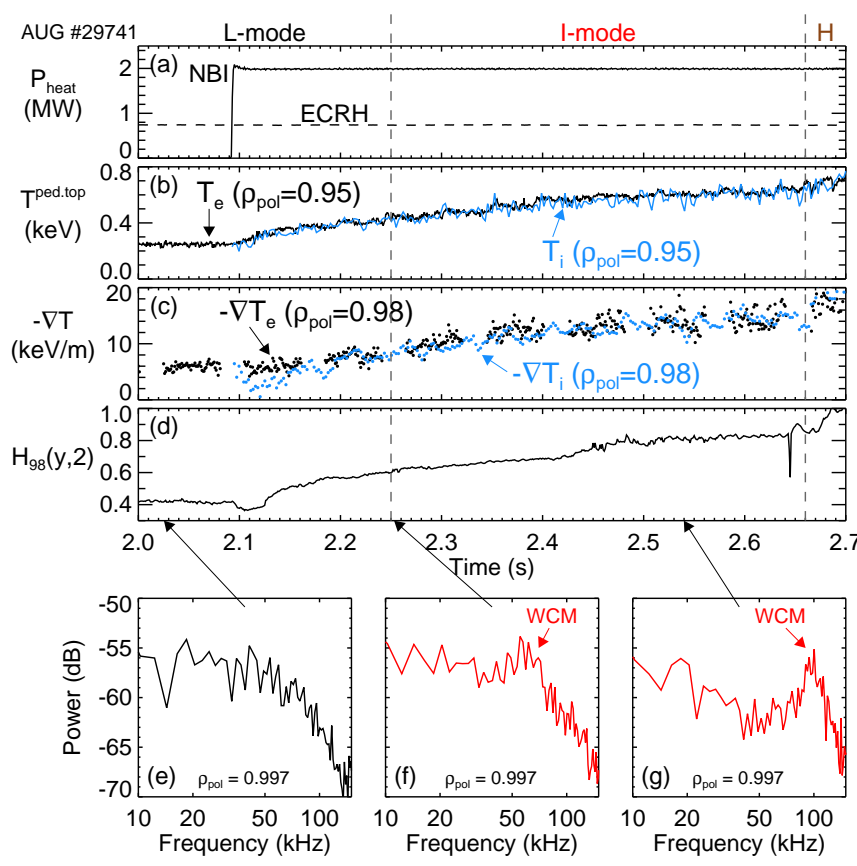

FIG. 1: Time traces going from L-mode through I-mode and into Hmode (indicated above) of (a) heating power, (b) pedestal top electron and ion temperatures, (c) edge electron and ion temperature gradients, and (d) confinement factor $H_{98}(y, 2)$. (e-g) Density turbulence spectra measured at the times indicated by the arrows show the appearance of the WCM in I-mode.

frequency and dominates the spectrum $(\mathrm{g})$. The pedestal top electron and ion temperatures (at $\rho_{\text {pol }}=0.95$ ) are depicted in (b), which increase with NBI and then throughout the whole Imode phase, which lasts for roughly $400 \mathrm{~ms}$. This is more than 4 energy confinement times $\left(\tau_{\mathrm{E}} \approx 94 \mathrm{~ms}\right)$, which shows that the I-mode is a slowly evolving process under fixed conditions at fixed heating input power. Stationary I-modes are obtained when the heating power is adjusted such that the confinement improvement factor $H_{98}(y, 2)$ [29] (cf Fig. 1(d)) remains below about 0.8 . As yet, such a situation has only been achieved with ECRH. Both electron and ion temperature gradients in the plasma edge at $\rho_{\mathrm{pol}}=0.98$ increase continuously during I-mode (c), which will be important later. $T_{i}$ is measured by charge exchange recombination spectroscopy on a beam of the neutral beam injection system [30]. In Fig. 1(d), $H_{98}(y, 2)$, which is 1 in $\mathrm{H}$-mode and up to 0.6 in L-mode, increases from 0.60 to 0.85 , which clearly shows the improved confinement in I-mode over L-mode.

During the evolution of the I-mode, both $\nabla T$ and $H_{98}(y, 2)$ increase, which is accompanied by a deepening of the radial profile of the perpendicular propagation velocity of the turbulence $v_{\perp}$, shown in Fig. 2. The bar in the lower left indicates the radial uncertainty in the density profile used to localize the Doppler reflectometer measurement positions, which affects all profiles equally. The relative uncertainties obtained with the beam-tracing code TorbeAm [31] are shown at each data point and correspond to roughly $\Delta R=2 \mathrm{~mm}$. This small ra-

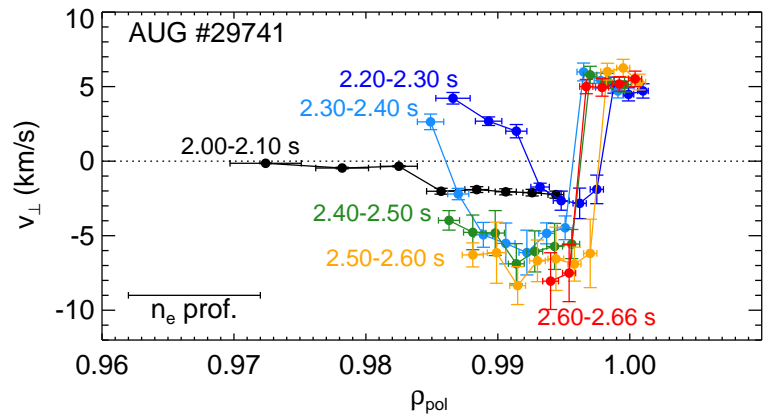

FIG. 2: The radial profile of the perpendicular propagation velocity of density fluctuations deepens with improving I-mode confinement (cf Fig. 1). In the lower left, the uncertainty of the density profile is indicated.

dial uncertainty is due to the fact that measurements are taken in the pedestal region with significant density gradient [32]. In the L-mode phase of the discharge $(2.00-2.10 \mathrm{~s})$, the $\mathrm{v}_{\perp}$ profile is rather flat and exhibits a shallow well at $\rho_{\text {pol }} \approx 0.99$ with a depth of $\mathrm{v}_{\perp} \approx-2 \mathrm{~km} / \mathrm{s}$. The radial position of the edge $\mathrm{v}_{\perp}$ well in AUG is generally at $\rho_{\text {pol }} \approx 0.99$ [33-35]. From 2.2 to $2.6 \mathrm{~s}$ two effects can be observed. First, the $\mathrm{v}_{\perp}$-well shifts outward to $\rho_{\text {pol }}=0.996(2.20-2.30 \mathrm{~s})$ and then inward as the well deepens at $\rho_{\text {pol }}=0.992$. Second, the rotation velocity of the plasma increases by a factor four from $-2 \mathrm{~km} / \mathrm{s}$ to about $-8 \mathrm{~km} / \mathrm{s}$, when the transition into H-mode takes place. Hence, there is a correlation between improved confinement and $\mathrm{v}_{\perp}$ well depth in the plasma edge, which was shown to be related to the neoclassical radial electric field dominated by the ion pressure gradient [33]. This is underlined by the fact that the measurements in Fig. 2 at $\rho_{\text {pol }}=0.99$ ( $\mathrm{v}_{\perp}$-minimum) agree with measurements from CXRS under the assumption that the phase velocity of the turbulence $v_{\mathrm{ph}}$ is small compared to the $E \times B$-velocity [27]. The wavenumber of the WCM can be estimated by $k_{\mathrm{WCM}}=2 \pi f_{\mathrm{WCM}} / \mathrm{v}_{\perp}$ to roughly $k_{\mathrm{WCM}} \approx 1.0-$ $1.5 \mathrm{~cm}^{-1}$, which is comparable to values found at Alcator CMod [7] and another AUG discharge [8]. It is important to note that the measurements reported in this letter are from the confinement region, in particular from the deep $\mathrm{v}_{\perp}$-well region. They are not SOL observations, which are known to show strong intermittency [18-23]. Assuming a constant $\mathrm{v}_{\perp}$-well width, a deeper $\mathrm{v}_{\perp}$-well translates into a stronger $\mathrm{v}_{\perp}$ shear. This can have substantial effect on turbulence [36], which is why it is essential to investigate edge turbulence in I-mode in detail.

Figure 3 compares two fluctuation amplitude time traces of $10 \mathrm{~ms}$ length measured with Doppler reflectometry during the L-mode (a) and the I-mode phase (b) of a plasma discharge comparable to the one shown in Fig. 1. The fluctuation amplitude is obtained directly from the amplitude signal of the heterodyne detection system [37]. The diagnostic measured at constant probing beam frequency, due to the unchanged density profile corresponding to the same radial position of $\rho_{\text {pol }} \approx 0.99$, which is close to the $\mathrm{v}_{\perp}$ minimum (cf Fig. 2). The L-mode signal in Fig. 3(a) has a roughly constant turbu- 

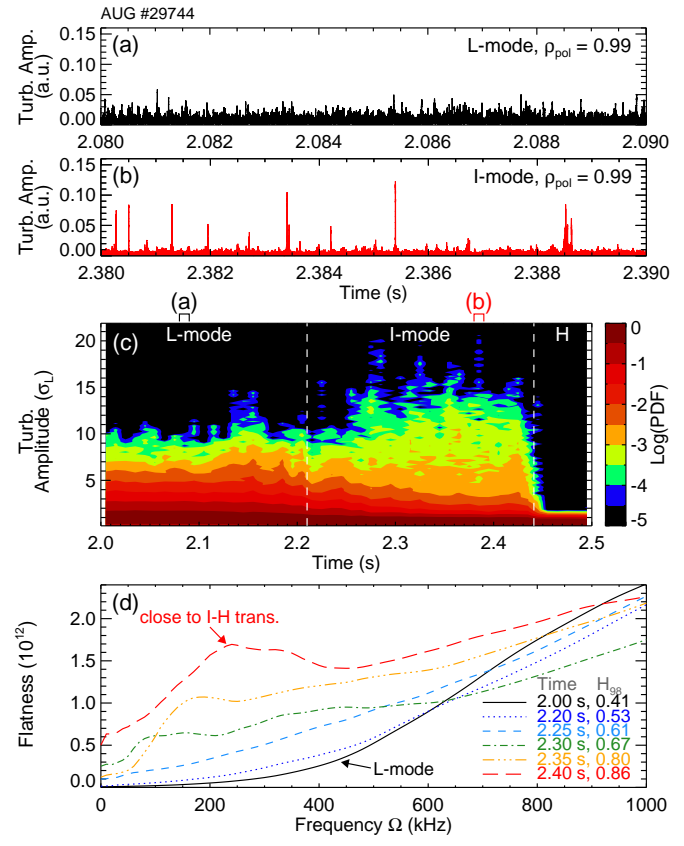

FIG. 3: Comparison of turbulence amplitude behavior in (a) L-mode and (b) I-mode. In I-mode, a low turbulence level and strong bursts are observed, while the L-mode exhibits a higher turbulence level. (c) The temporal evolution of the probability density function from L-mode through I-mode into H-mode. The time windows from (a) and (b) are indicated on top. (d) A gradual increase of flatness is observed in I-mode.

lence level with few extrema or spikes. In contrast, the I-mode signal (b) has a lower base turbulence level, but it exhibits strong irregularly spaced turbulence bursts, which are significantly stronger than any fluctuations in L-mode (a) or H-mode (not shown). These intermittent events last for $2-10 \mu \mathrm{s}$. In Fig. 3(c), the temporal evolution of the probability density function (PDF) is shown in color-coded contours from Lmode, through the I-mode into $\mathrm{H}$-mode. To make a meaningful comparison, the turbulence amplitudes have been normalized to the value of the L-mode standard deviation $\sigma_{\mathrm{L}}$. While the occurrence of smaller events $\left(<6 \sigma_{\mathrm{L}}\right)$ is reduced from Lmode to I-mode, the probability for large events $\left(>10 \sigma_{\mathrm{L}}\right)$ increases. It should be noted that the bursts in I-mode can have amplitudes of up to 20 standard deviations of the L-mode signal, which underlines that they are of particularly strong intensity.

To quantify the intermittency of the density fluctuation amplitude signals, the flatness is used. It is defined as $F(\Omega)=$ $\left.\left\langle\left(s_{\Omega}^{\mathrm{HP}}(t)\right)^{4}\right\rangle /\left\langle\left(s_{\Omega}^{\mathrm{HP}}(t)\right)^{2}\right)\right\rangle^{2}$, where $s_{\Omega}^{\mathrm{HP}}(t)$ is the high-pass filtered fluctuation amplitude time series above the frequency $\Omega$ and $\langle\cdot\rangle$ denotes ensemble averaging. It thus gives a measure of the kurtosis of a high-pass filtered signal normalized to its squared variance. The flatness $F$ in Fig. 3(d) can consequently be viewed as a measure of the intermittency as a function of the filter frequency, and hence, of the scale size [38, 39]. Clearly the flatness increases as the I-mode develops for frequencies $\Omega<600 \mathrm{kHz}$ up to a factor of 10 , and a peak appears
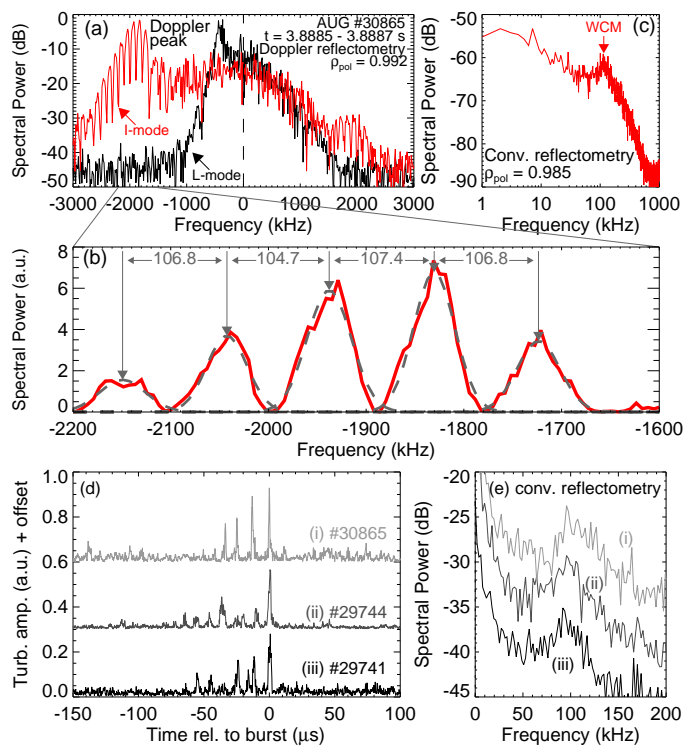

FIG. 4: (a) Doppler peak for an I-mode burst (red) at $f_{\mathrm{D}} \approx$ $-1900 \mathrm{kHz}$, quantized due to frequency modulation, compared to a general L-mode case (black). The frequency separation of individual components is visible in the zoom to the Doppler peak and on linear scale. (c) Power spectrum of a conventional reflectometer, showing the existence of the WCM at $f_{\mathrm{WCM}} \approx 115 \mathrm{kHz}$. (d) Examples from different plasma discharges showing the turbulence amplitude evolution leading up to the burst. The frequency of the precursor events corresponds to the WCM frequency, as depicted in (e).

around $200 \mathrm{kHz}$ for I-modes which are close to the I-H transition (red dashed). Note these frequencies correspond to the inverse of the time during which the structures are observed $(2-10 \mu \mathrm{s})$. At frequencies $\Omega>600 \mathrm{kHz}$, no clear trend is seen. Also note that the flatness increases continuously with confinement, which reflects the gradual development of the I-mode over several confinement times.

Since both the bursts and the WCM are observed in I-mode, a connection between them is now investigated. Figure 4(a) shows a frequency spectrum from the Doppler reflectometer heterodyne signal during an I-mode burst measured at $\rho_{\text {pol }}=$ 0.992 (red). The Doppler shift is at roughly $-1900 \mathrm{kHz}$. In contrast to the L-mode case (black), where a normal Doppler shifted peak is observed at roughly $-400 \mathrm{kHz}$, the peak in I-mode is quantized, i.e. it is spread into multiple narrow sub-peaks. The larger Doppler shift in I-mode compared to L-mode reflects the deeper $\mathrm{v}_{\perp}$ well (cf Fig. 2). A zoom of the I-mode Doppler peak on linear scale is shown in Fig. 4(b). The clear separation into individual components can be understood as follows: if the signal received by a Doppler reflectometer is amplitude modulated with a frequency $f_{\text {mod }}$, the Doppler shift will be discretized into individual components, separated in frequency by $f_{\text {mod }}$. While a sinusoidal amplitude modulation results in only two sidebands, a non-sinusoidal modulation, as presented later in Fig. 4(d), can result in several sidebands. The grey dashed lines show Gaussian fits to the individual components, with the frequency spacing be- 
tween the components indicated in the upper part of the panel. The mean frequency difference between individual peaks is $f=106.4 \pm 1.2 \mathrm{kHz}$. This value compares well with the frequency of the WCM measured with conventional reflectometry of $f_{\mathrm{WCM}} \approx 115 \mathrm{kHz}$ (cf Fig. 4(c)). The small frequency discrepancy is due to slightly different radial measurement locations of the two diagnostics (indicated in the figure), where $\mathrm{v}_{\perp}$ differs slightly. The peak splitting effect is observed in Imodes with good energy confinement $\left(H_{98}(y, 2)>0.75\right)$, and thus shows the connection between WCM and the observed high-amplitude bursts. The observed events are usually accompanied by smaller density perturbations, leading up to the burst. This is visualized in Fig. 4(d), where small turbulence bursts are seen before the final burst. The temporal separation of the precursor events $\Delta t$ corresponds to the WCM frequency $f_{\mathrm{WCM}} \approx 1 / \Delta t$, depicted from conventional reflectometry measurements in (e), which shows again the connection between bursts and WCM. This effect is generally observed in I-mode plasmas, the whole succession of events takes around $40-$ $100 \mu \mathrm{s}$. This turbulence amplitude modulation by the WCM leads to the peak splitting effect in Fig. 4(a). It does not seem to be due to a simple beam path modulation by the WCM, which - in contrast to Fig. 4(d) - would not strongly affect the turbulence amplitude measurement. The above shows a correlation between density bursts and WCM. A proof of causality would involve the treatment of energy transfer, which cannot be measured with the present confinement region turbulence diagnostics on AUG.

As stated above and in Ref. [8], the conventional reflectometry measurements show that the WCM exists at a wavenumber of $k_{\mathrm{WCM}}=2 \pi f_{\mathrm{WCM}} / \mathrm{v}_{\perp} \approx 1.0-1.5 \mathrm{~cm}^{-1}$, while the Doppler reflectometer measurements presented are at $k_{\perp}=$ $11-13 \mathrm{~cm}^{-1}$. In fact, even including beam wavefront and cutoff-layer curvatures $[40,41]$, the conventional reflectometer measures wavenumbers in the range of $k_{\perp}=0-2 \mathrm{~cm}^{-1}$. These scales are substantially different and the question arises why measurements at high $k_{\perp}$ observe effects which are obviously related to the WCM. A possible explanation is that the WCM modulates the amplitude of small-scale structures. Thus, the turbulence level observed by Doppler reflectometry should be modulated with $f_{\mathrm{WCM}}$ as observed in Fig. 4(d), and although it is not the WCM which is directly observed, WCM features like its frequency will be observed even at higher $k_{\perp}$. A somewhat comparable but more interesting consideration is that the waveform of the WCM might become asymmetric, so-called wave steepening, and thus would contain higher $k_{\perp}$. No clear conclusion can be drawn at this point other than there is a clear influence of the WCM on comparably small-scale turbulence which leads to the pronounced turbulence bursts reported here.

All discharges presented here exhibit a GAM. Since it is the most stationary, for discharge AUG \#30865 (Fig. 4(a)), a coupling between GAM and WCM has been documented at a radial position of $\rho_{\text {pol }} \approx 0.99$ [8].

It is important to note that the intermittent events do not exhibit features in common with the typical edge-localized
MHD instability (ELM), for a review see Ref. [42]. Type I and type III ELMs usually occur periodically, furthermore type I ELMs erode both density and temperature pedestals, which manifests in a strong energy loss of the plasma. Neither of these effects is observed in I-mode. Furthermore, I-modes are in general peeling-ballooning stable $[4,5]$. Type II (grassy) ELMs can be excluded because the plasmas reported here are not strongly shaped [42].

Moreover, while the observed bursts do not appear in the electron temperature, they are visible in conventional reflectometry, bolometry, and magnetic probe signals [2, 43], which points to a dominance of density fluctuations and their existence at perpendicular wavenumbers other than the relatively high $k_{\perp}$ structures reported on in this letter. The detailed analysis of other diagnostics is out of the scope of this letter and left for future work.

The large amplitudes and localization in time of the events reported in this letter are very pronounced. There are shock models, as e.g. discussed in Ref. [44, 45], which show similarities with the local plasma properties in I-mode. In a simple non-linear electrostatic drift-wave model [46] including both density and temperature gradients the evolution of the plasma potential is given by

$$
\frac{e}{T} \frac{\partial \tilde{\phi}}{\partial t}=\frac{1}{B} \frac{\partial \tilde{\phi}}{\partial y}\left(\frac{1}{n} \frac{\partial n}{\partial x}-\frac{1}{T} \frac{\partial T}{\partial x} \frac{e \tilde{\phi}}{T}\right),
$$

where $e$ is the elementary charge, $\tilde{\phi}$ is the perturbed plasma potential, $n$ and $T$ are the background density and temperature profiles, and $x$ and $y$ correspond to the radial and poloidal directions, respectively. The first term on the right-hand side (rhs) is a turbulent drive. The second term can be written as

$$
-\frac{\partial T / \partial x}{T} \tilde{\phi} \frac{\partial \tilde{\phi}}{\partial y}
$$

and then has the form of the non-linearity appearing in the Korteweg-de-Vries (KdV) and Burgers equations known to be responsible for intermittency in $1 \mathrm{D}$ systems [47]. Whereas the Burgers equation describes shocks, the $\mathrm{KdV}$ equation is used to model solitons. Therefore shocks and solitons are driven by the same nonlinearity, but differ by the dominant damping which is dispersive for solitons and dissipative for shocks. In fact, (1) describes the growth of so-called shock-like drift waves [46]. The energetic behavior of such systems is characterized by a strong non-linear energy transfer to high frequencies, which manifests itself in an increase of short-duration events, as is observed here. The non-linear energy is driven by the term (2), which is proportional to the temperature gradient, shown to evolve steadily during I-mode (cf Fig. 1(d)). In the simple model [46] only poloidal propagation has been considered, raising the question whether shock-like drift waves can be generated in the 2D system of a fusion plasma. Generalized to pressure fluctuations $\tilde{p}$ the non-linear part of drift wave turbulence is given by

$$
\frac{\partial}{\partial t} \tilde{p}=\{\tilde{\phi}, p\}=\{\tilde{\phi}, \bar{p}\}+\{\tilde{\phi}, \tilde{p}\}
$$


Here, the Poisson bracket corresponds to $\{\tilde{\phi}, \cdot\}=\partial_{x} \tilde{\phi} \partial_{y}-$ $\partial_{y} \tilde{\phi} \partial_{x}$, and $\bar{p}$ and $\tilde{p}$ are background and fluctuating pressure, respectively. The term $\{\tilde{\phi}, \bar{p}\}$ is the turbulence drive and $\{\tilde{\phi}, \tilde{p}\}$ the non-linear interaction, which can be written

$$
\{\tilde{\phi}, \tilde{p}\}=\bar{T}\{\tilde{\phi}, \tilde{n}\}+\tilde{n}\{\tilde{\phi}, \bar{T}\}+\bar{n}\{\tilde{\phi}, \tilde{T}\}+\tilde{T}\{\tilde{\phi}, \bar{n}\},
$$

where the second term on the rhs corresponds to (2). Generally, the second and fourth terms on the rhs are neglected because they are of second order. If, for example, $k_{x}=0$, the first and third terms vanish, resulting in intermittent solitons or shocks. This is not possible in a $2 \mathrm{D}$ fusion plasma. Nevertheless, in the case of the I-mode plasmas reported here, the second term could be important and be in competition with the first and third terms due to coexistence of the strong background temperature gradient and the strong turbulence amplitudes, as shown in the experimental part of this letter, which could then lead to the generation of the solitary structures reported. The solitary waveform points to stronger dispersive effects compared to dissipation, which would lead to a more sawtooth-like structure.

Comparing the three regimes (L-mode, I-mode and $\mathrm{H}$ mode) the relative contribution of the second term on the rhs of (1) is strongest in I-mode. Moreover, assuming adiabatic electrons, $\tilde{n} \propto \tilde{\phi}$, it is seen from (1) that the temperature gradient can drive strong fluctuations in density, as observed experimentally in this letter. In the work reported here, clearly the bursty character and intermittency in the density turbulence (cf Fig. 3) increase together with the temperature gradient during the I-mode phase of the discharge (cf Fig. 1(c)). Since strong intermittent density bursts can be driven by the temperature gradient, it could be speculated that the density profile growth is limited through the bursts, which may explain the lack of a density pedestal in I-mode. However, it has to be noted that in order to prove this statement, measurements at different $k_{\perp}$ and assumptions on related particle transport would be necessary, which is outside the scope of this letter.

Another explanation of the observed intermittency can be provided by Ref. [48]. The non-local interaction in wavenumber space between zonal flows and drift-waves can lead to a Burgers-type equation and again solitary structures in $k_{r^{-}}$ space. GAMs, which are finite frequency zonal flows and generally observed to be coupled to the WCM $[7,8]$, can lead to similar processes. There is of course the possibility that the observed bursts are due to a symbiotic occurrence of both effects. Analog to the sandpile model bursts are generated by the GAM-drift-wave interaction [48], which are afterward amplified by the KdV-nonlinearity described above.

In summary, this letter reports on the observation of strongly intermittent turbulent density bursts in the confinement region of I-mode plasmas in the ASDEX Upgrade tokamak. It has been shown that the increase in confinement in I-mode is correlated with a deepening of the edge $\mathrm{v}_{\perp}$ well. In this context, the I-mode is situated in parameter space between L-mode and $\mathrm{H}$-mode in both confinement quality and perpendicular velocity shear. While low-amplitude density fluctuations decrease from L- to I-mode, strong density turbulence bursts develop during the I-mode. These density turbulence bursts are intermittent, leading to a strongly tailed PDF. Furthermore, the observed bursts are connected to the weakly coherent mode, which is an intrinsic feature of the I-mode in all experiments to date, underlining the importance of the observed bursts. The generation mechanisms suggested here involve the temperature gradient and GAM-drift-wave interactions as central elements.

Fruitful discussions with S. Freethy, W. Suttrop, and J. Vicente are gratefully acknowledged. This work was partly performed in the framework of the Helmholtz Virtual Institute on plasma dynamical processes and turbulence using advanced microwave diagnostics (VH-VI-526) and within the framework of the EUROfusion Consortium and has received funding from the Euratom research and training programme 20142018 under grant agreement No 633053. The views and opinions expressed herein do not necessarily reflect those of the European Commission.

* Electronic address: tim.happel@ipp.mpg.de

[1] F. Wagner et al., Phys. Rev. Lett. 49, 1408 (1982).

[2] F. Ryter et al., Plasma Phys. Control. Fusion 40, 725 (1998).

[3] D. G. Whyte et al., Nucl. Fusion 50, 105005 (2010).

[4] J. Hughes et al., Nucl. Fusion 53, 043016 (2013).

[5] J. R. Walk et al., Phys. Plasmas 21, 056103 (2014).

[6] A. E. Hubbard et al., Phys. Plasmas 18, 056115 (2011).

[7] I. Cziegler et al., Physics of Plasmas 20, 055904 (2013).

[8] P. Manz et al., Nucl. Fusion 55, 083004 (2015).

[9] J. Paret and P. Tabeling, Phys. Fluids 10, 3126 (1998).

[10] Y. Jun and X. L. Wu, Phys. Rev. E 72, 035302 (2005).

[11] R. T. Cerbus and W. I. Goldburg, Phys. Fluids 25, 105111 (2013).

[12] P. Bak, C. Tang, and K. Wiesenfeld, Phys. Rev. Lett. 59, 381 (1987).

[13] R. Jha et al., Phys. Rev. Lett. 69, 1375 (1992).

[14] R. A. Moyer et al., Plasma Phys. Control. Fusion 38, 1273 (1996).

[15] T. Rhodes et al., Phys. Lett. A 253, 181 (1999).

[16] G. Y. Antar et al., Phys. Rev. Lett. 87, 065001 (2001).

[17] R. Sánchez, B. P. van Milligen, D. E. Newman, and B. A. Carreras, Phys. Rev. Lett. 90, 185005 (2003).

[18] G. Y. Antar et al., Phys. Plasmas 10, 419 (2003).

[19] T. Happel et al., Phys. Rev. Lett. 102, 255001 (2009).

[20] B. Nold et al., Plasma Phys. Control. Fusion 52, 065005 (2010).

[21] D. A. D'Ippolito, J. R. Myra, and S. J. Zweben, Phys. Plasmas 18, 060501 (2011).

[22] O. E. Garcia, Phys. Rev. Lett. 108, 265001 (2012).

[23] O. E. Garcia et al., J. Nucl. Mat. 438, S180 (2013), proceedings of the 20th International Conference on Plasma-Surface Interactions in Controlled Fusion Devices.

[24] A. Herrmann and O. Gruber, Fusion Sci. Technol. 44, 569 (2003).

[25] T. Happel et al., The optimized steerable W-band Doppler reflectometer on ASDEX Upgrade: possibilities and issues (Proc. $11^{\text {th }}$ International Reflectometry Workshop, Palaiseau, France, 2013).

[26] R. Sabot, P. Hennequin, and L. Colas, Fusion Sci. Technol. 56, 
1253 (2009).

[27] F. Ryter and et al., Nucl. Fusion (2016), in preparation.

[28] L. Cupido et al., Rev. Sci. Instrum. 77, 10E915 (2006).

[29] ITER Physics Basis Editors, ITER Physics Expert Group Chairs and Co-Chairs, ITER Joint Central Team, and Physics Integration Unit, Nucl. Fusion 39, 2137 (1999).

[30] E. Viezzer et al., Rev. Sci. Instrum. 83, (2012).

[31] E. Poli, A. G. Peeters, and G. V. Pereverzev, Comput. Phys. Commun. 136, 90 (2001).

[32] H.-J. Hartfuß and T. Geist, Fusion Plasma Diagnostics with mm-Waves (Wiley-VCH, Boschstr. 12, 69469 Weinheim, Germany, 2013).

[33] E. Viezzer et al., Nucl. Fusion 53, 053005 (2013).

[34] T. Happel et al., Phys. Plasmas 22, 032503 (2015).

[35] G. D. Conway et al., Plasma Phys. Control. Fusion 57, 014035 (2015).

[36] K. H. Burrell, Phys. Plasmas 4, 1499 (1997).

[37] H. J. Hartfuss, T. Geist, and M. Hirsch, Plasma Phys. Control. Fusion 39, 1693 (1997).
[38] U. Frisch, Turbulence (University of Cambridge, Trumpington Street, Cambridge, United Kingdom, 1995).

[39] N. Mahdizadeh et al., Phys. Plasmas 11, 3932 (2004).

[40] Y. Lin, R. Nazikian, J. H. Irby, and E. S. Marmar, Plasma Phys. Control. Fusion 43, L1 (2001).

[41] M. Hirsch et al., Plasma Phys. Control. Fusion 43, 1641 (2001).

[42] H. Zohm, Plasma Phys. Control. Fusion 38, 105 (1996).

[43] T. Happel et al., Plasma Phys. Control. Fusion (2016), in preparation.

[44] Y. B. Zel'dovich and Y. P. Raizer, Physics of Shock Waves and High-Temperature Hydrodynamic Phenomena (Academic Press Inc., New York and London, 1967).

[45] A. Balogh and R. A. Treumann, Physics of Collisionless Shocks (Springer, New York, 2013).

[46] H. Tasso, Phys. Lett. A 24, 618 (1967).

[47] E. Balkovsky, G. Falkovich, I. Kolokolov, and V. Lebedev, Phys. Rev. Lett. 78, 1452 (1997).

[48] P. H. Diamond and M. Malkov, Phys. Scr. T98, 63 (2002). 